\title{
SURVEY COORDINATES \\ OF THE BOOSTER-TO-AGS TRANSPORT LINE
}

BOOSTER TECHNICAL NOTE

NO. 221

F. X. Karl and M. A. Goldman

February 17, 1993

ALTERNATING GRADIENT SYNCHROTRON DEPARTMENT

BROOKHAVEN NATIONAL LABORATORY

UPTON, NEW YORK 11973 
SURVEY COORDINATES OF THE

BOOSTER-TO-AGS TRANSPORT LINE

\author{
F.X. Karl, M.A. Goldman \\ January 27,1993
}

The Booster to AGS transport line [BTA line], used to inject protons and heavy ions from the Booster into the AGS, was surveyed and aligned during october and December 1990. The surveyors were F. Karl, D. Kazmark, Jr. and J. Roecklein, of the BNL/AGS Survey Group.

Survey reference data is stored in File BTA7390.300 of the BNL/AGS Survey Group. Drawings describing locations of reference points of beam components are also available on file.

The transport line is described in section 5 of the AGS Booster project's Booster Design Manual, Revision 1, October 1988. The layout of the transfer line magnets is given in AGS Booster drawing D37-M-0413-5H. [Booster To AGS Transfer Line, Component Stand Locations, J. A. Scheblein; June 14, 1990]. The drawing numbers of individual magnet components of the transfer line are listed in that drawing, together with a plan view layout of the line, showing defining dimensions of the line. The line components were aligned to the positions and spacings called out in that drawing, during the survey of 1990 . 
The survey was referenced to the survey pillar monuments "L" and "LA" in the AGS ring. These monument stations were used as fixed control references for the BTA line survey. Their horizontal coordinates were measured in the 1987 horizontal survey of the AGS ring [1], and are:

AGS Inch Coordinates

EAST NORTH

AGS Pillar Monument " L " $\quad 2469.198 \quad 14582.679$ (Survey Point 100)

AGS Pillar Monument " LA " $1953.146 \quad 13336.891$ (Survey Point 99)

[ The AGS machine center has AGS Inch coordinates: EAST 6942.600, NORTH 12000.000 ].

The AGS Inch Coordinates of transport line components, coordinate increments between successive components, and distances between successive components are listed in Table 1 . The transport line starts at the AGS Injection Point, survey Point 24, with coordinates: EAST 2381.8431, NORTH 15685.661.

The vertical focus quadrupole magnet QV1 was placed 2" downstream from the position specified in the Booster Design Manual, to avoid mechanical interferences. For the front end septa and magnets QHI through DH4 the magnet placements are the same in the Booster Design Manual and drawing D37-M-0413-5H. Magnets QV9 through QV15 were installed at the locations called out in that drawing, and differ from those in the Design Manual. 
Elevation views, showing layout of the line components are given in AGS Booster drawings: D37-M-0391-5C, D37-M-0392-5B, D37-M-0393-5A, Booster To AGS Transfer Line, J.A. Scheblein, January 15, 1991.

AGS drawings relating to the Booster ejection septa are: D14-1510-M-5A, Building 914 Booster Ejection Septum Shielding Assembly, April 1, 1991; D37-M-0391-5A, Booster To AGS Transfer Line Ejection Septum to Q7 Assembly, January 15,1991; Booster To AGS Transfer Line Q12 to AGS Injection Septum Assembly, January 15, 1991 .

AGS drawings relating to the transfer line pickup electrodes are: D37-M-0265-4C, Pickup Electrode Assembly End Plate, July 26, 1989; D37-M-0501-4A, Booster To AGS Transfer Line Q5 \& Q14 PUE Chamber Weldment Assembly, September 4, 1990; D36-M1242-5E, AGS Booster QUAD/PUE Vacuum Shell WeldmentFebruary 15, 1989; D37-M-0586-4A, Booster-AGS Transfer Line PUE Chamber Rework Q11, Q12\& Q13 Assembly, November 7, 1990.

The PUE center for quadrupole magnet QV5 is located 14.473" downstream of the center point of that magnet. The center of the PUE for each of the quadrupole magnets: QV11, QH12, QV13, QH14 is located 14.473" upstream of the center of the corresponding magnet. 
Table 1. BTA Line coordinates

\begin{tabular}{|c|c|c|c|c|c|c|}
\hline \multicolumn{2}{|c|}{$\begin{array}{l}\text { BTA LINE } \\
\text { COMPONENTS }\end{array}$} & \multicolumn{2}{|c|}{$\begin{array}{l}\text { AGS Inch } \\
\text { Coordinates }\end{array}$} & \multicolumn{2}{|c|}{$\begin{array}{c}\text { Coordinate Increment } \\
\text { [Inches] }\end{array}$} & \multirow[t]{2}{*}{$\begin{array}{l}\text { Distance } \\
\text { Increment } \\
\text { [Inches] }\end{array}$} \\
\hline $\begin{array}{l}\text { SURVEY } \\
\text { POINT }\end{array}$ & NAME & & & & & \\
\hline PUINI & INAIIL & EAST & NORTH & DELTA E & DELTA $\mathbf{N}$ & $S$ DIFF \\
\hline \multirow[t]{4}{*}{24} & $\begin{array}{l}\text { I.P. } \\
\text { (AGS Inje }\end{array}$ & $\begin{array}{l}2381.8431 \\
\text { ction Point) }\end{array}$ & 15685.661 & & & \\
\hline & SEPTUM & 2379.9487 & 15711.5102 & & & \\
\hline & SEPTUM & 2404.7712 & 15616.0374 & 24.8225 & -95.4728 & 98.6469 \\
\hline & QV1 & 2436.8441 & 15518.641 & 32.0729 & -97.3964 & 102.5414 \\
\hline 2 & DH1 & 2448.5336 & 15483.1451 & 11.6895 & -35.4959 & 37.3712 \\
\hline \multirow[t]{2}{*}{3} & QH2A & 2458.386 & 15449.1082 & 09.8524 & -34.0369 & 35.4342 \\
\hline & QH2B & 2466.0487 & 15422.6358 & 07.6627 & -26.4724 & 27.5591 \\
\hline 4 & QV3 & 2480.7655 & 15371.794 & 14.7168 & -50.8418 & 52.9289 \\
\hline 5 & STRIPPER & 2487.8809 & 15347.2126 & 07.1154 & -24.5814 & 25.5905 \\
\hline 6 & $\mathrm{QH} 4$ & 2501.5644 & 15299.9406 & 13.6835 & -47.272 & 49.2126 \\
\hline 7 & DH2 & 2523.0049 & 15225.8703 & 21.4405 & -74.0703 & 77.1110 \\
\hline 8 & QV5 & 2523.4486 & 15152.6977 & 00.4437 & -73.1726 & 73.1740 \\
\hline 9 & DH3 & 2524.0535 & 15052.9457 & 00.6049 & -99.752 & 99.7538 \\
\hline 10 & QH6 & 2503.5128 & 14978.6209 & -20.5407 & -74.3248 & 77.1109 \\
\hline 11 & QV7 & 2483.5868 & 14906.5205 & -19.926 & -72.1004 & 74.8032 \\
\hline 12 & QH8 & 2389.2008 & 14564.9922 & -94.386 & -341.528 & 354.3308 \\
\hline 13 & DH 4 & 2376.0916 & 14517.5578 & -13.1092 & -47.4344 & 49.2125 \\
\hline 14 & QV9 & 2350.2192 & 14429.8063 & -25.8724 & -87.7515 & 91.4861 \\
\hline
\end{tabular}




$\begin{array}{lllllll}15 & \text { QH10 } & 2317.0444 & 14317.2874 & -33.1748 & -112.519 & 117.3076 \\ 16 & \text { QV11 } & 2243.363 & 14067.3826 & -73.6814 & -249.905 & 260.5405 \\ 17 & \text { QH12 } & 2192.141 & 13893.6531 & -51.222 & -173.729 & 181.1233 \\ 18 & \text { QV13 } & 2132.3759 & 13690.9478 & -59.7651 & -202.705 & 211.3322 \\ & \text { QH14 } & 2108.0537 & 13608.4544 & -24.3222 & -82.4935 & 86.0043 \\ 20 & \text { DH5 } & 2092.6908 & 13556.3479 & -15.0976 & -51.2067 & 53.38599 \\ 21 & \text { QV15 } & 2078.3562 & 13458.813 & -14.3346 & -97.5349 & 98.58264 \\ & \text { SEPTB1 } & 2062.6363 & 13351.8517 & -15.7199 & -106.961 & 108.1103\end{array}$

BTA LINE

DIAGNOSTIC AND TRIM COMPONENTS

\begin{tabular}{|c|c|c|c|c|}
\hline $\begin{array}{l}\text { PUE V.046 } 2523.5364 \\
\text { (Pickup Electrode) }\end{array}$ & 15138.2250 & $\begin{array}{l}0.0878 \\
\text { (From }\end{array}$ & $Q V 5)^{-14.4727}$ & $\begin{array}{c}14.473 \\
\text { (From QV5) }\end{array}$ \\
\hline $\begin{array}{lc}\text { XF059 } & 2495.9543 \\
\text { (Beam current Monitor }\end{array}$ & $\begin{array}{l}14951.2712 \\
\text { Transformer) }\end{array}$ & $\begin{array}{r}-7.5585 \\
\text { (From }\end{array}$ & $\begin{array}{l}-27.3498 \\
\mathrm{QH} 6)\end{array}$ & $\begin{array}{l}28.375 \\
\text { (From QH6) }\end{array}$ \\
\hline $\begin{array}{l}\text { MW060 } 2491.0263 \\
\text { (Profile Monitor, Mult }\end{array}$ & $\begin{array}{l}14933.4396 \\
\text { tiwire) }\end{array}$ & $\begin{array}{c}-12.4865 \\
\text { (From }\end{array}$ & $Q H 6)^{-45.1813}$ & $\begin{array}{c}46.875 \\
(\text { From QH6) }\end{array}$ \\
\hline $\begin{array}{l}\text { MW125 } 2258.2638 \\
\text { (Profile Monitor, Mult }\end{array}$ & $\begin{array}{l}14117.9215 \\
\text { tiwire) }\end{array}$ & $\begin{array}{c}-58.7806 \\
\text { (From }\end{array}$ & $\begin{array}{l}-199.3659 \\
Q H 10)\end{array}$ & $\begin{array}{l}207.8505 \\
\text { (From QH10) }\end{array}$ \\
\hline $\begin{array}{l}\text { DH127 } 2252.1129 \\
\text { (Trim Dipole) }\end{array}$ & 14097.0594 & $\begin{array}{r}-64.9315 \\
\text { (From }\end{array}$ & $\begin{array}{l}-220.2280 \\
Q H 10)\end{array}$ & $\begin{array}{l}229.6005 \\
(\text { From QH10) }\end{array}$ \\
\hline PUE_V.129 2247.4560 & 14081.2647 & $\begin{array}{l}4.0930 \\
\text { (From }\end{array}$ & $\begin{array}{l}13.8821 \\
Q V 11)\end{array}$ & $\begin{array}{l}-14.473 \\
\text { (From QV11) }\end{array}$ \\
\hline $\begin{array}{l}\text { DV141 } 2200.8909 \\
\text { (Trim Dipole) }\end{array}$ & 13923.3306 & $\begin{array}{r}-42.4721 \\
\text { (From }\end{array}$ & $\begin{array}{l}-144.0520 \\
Q V 11)\end{array}$ & $\begin{array}{l}150.1832 \\
\text { (From QV11) }\end{array}$ \\
\hline
\end{tabular}




$$
\begin{aligned}
& \begin{array}{llccc}
\text { PUE_H.143 2196.2340 } & 13907.5353 & 4.0930 & 13.8822 & -14.473 \\
& & (\text { From QH12) } & \text { (From QH12) }
\end{array}
\end{aligned}
$$

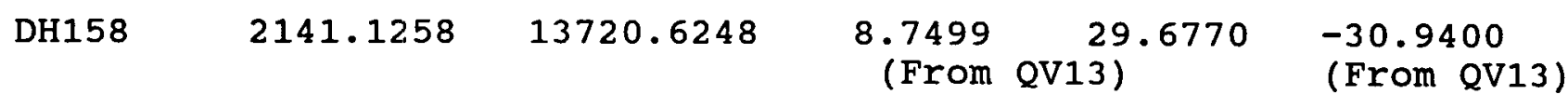

$$
\begin{aligned}
& \begin{array}{cccccc}
\text { PUE_V.160 } & 2136.4689 & 13704.8300 & 4.090 & 13.8822 & \text { (From QV13) } \\
\text { (From QV13) }
\end{array}
\end{aligned}
$$

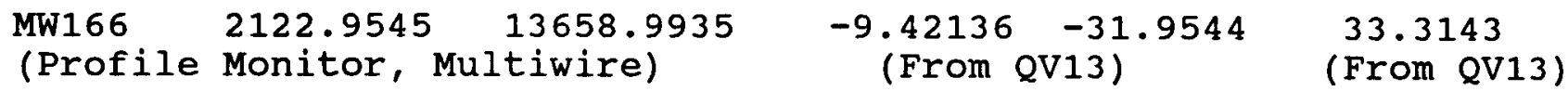

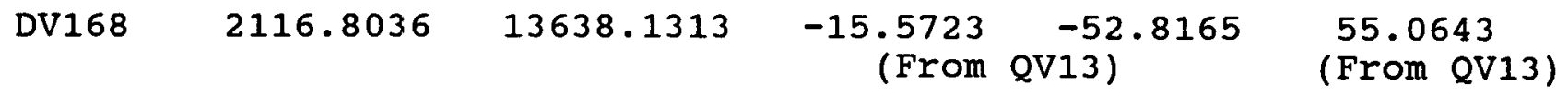

$$
\begin{aligned}
& \begin{array}{lllll}
\text { PUE_H.170 } 2112.1467 & 13622.3366 & 4.0930 & 13.8822 & -14.473 \\
& & & \text { (From QH14) } & \text { (From QH14) }
\end{array}
\end{aligned}
$$

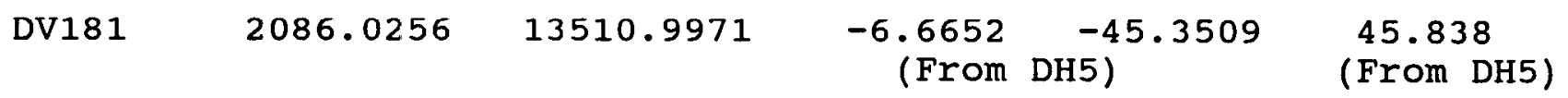

$$
\begin{aligned}
& \begin{array}{cccccc}
\text { XF183 } & 2083.1538 & 13491.4569 & -9.5370 & -64.8910 & 65.588 \\
\text { (Beam Current Monitor, Transformer) } & \text { (From DH5) } & \text { (From DH5) }
\end{array}
\end{aligned}
$$

In this table: listed quadrupole magnet coordinates are those of the quadrupole center; the dipole magnet coordinates are those of the dipole magnet apex; the PUE coordinates are those of the PUE center.

When BTA transport line components are listed as elements in the modular accelerator design (MAD) beam-optical transport accelerator code, their designation nomenclature is that of the present note, prefaced by "ABI." . For example, the horizontally focusing dipole magnet DH3 would be listed as ABI.DH3 . 
Table 1A.

\begin{tabular}{|c|c|c|c|c|c|c|}
\hline \multicolumn{2}{|c|}{$\begin{array}{l}\text { BTA LINE } \\
\text { COMPONENTS }\end{array}$} & \multicolumn{2}{|c|}{$\begin{array}{l}\text { AGS Meter } \\
\text { Coordinates }\end{array}$} & \multicolumn{2}{|c|}{$\begin{array}{c}\text { Coordinate Increment } \\
\text { [Meters] }\end{array}$} & \multirow{3}{*}{$\begin{array}{l}\text { Distance } \\
\text { Increment } \\
\text { [Meters] } \\
\text { S DIFF }\end{array}$} \\
\hline \multicolumn{6}{|l|}{ SURVEY } & \\
\hline POINT & NAME & EAST & NORTH & DELTA E & DELTA $N$ & \\
\hline \multirow[t]{4}{*}{24} & $\begin{array}{l}\text { I.P. } \\
\text { (AGS Inje }\end{array}$ & $\begin{array}{l}60.49881 \\
\text { ion Point) }\end{array}$ & 398.41579 & & & \\
\hline & SEPTUM & 60.45070 & 399.07236 & & & \\
\hline & SEPTUM & 61.08119 & 396.64735 & 0.63049 & -2.42501 & 2.50563 \\
\hline & QV1 & 61.87996 & 394.22173 & 0.79877 & -2.42562 & 2.55375 \\
\hline 2 & DH1 & 62.19275 & 393.27189 & 0.31279 & -0.94985 & 1.00003 \\
\hline \multirow[t]{2}{*}{3} & $\mathrm{QH} 2 \mathrm{~A}$ & 62.44300 & 392.40735 & 0.25025 & -0.86454 & 0.90003 \\
\hline & $\mathrm{QH} 2 \mathrm{~B}$ & 62.63764 & 391.73495 & 0.19463 & -0.67240 & 0.70000 \\
\hline 4 & QV3 & 63.01144 & 390.44357 & 0.37381 & -1.29138 & 1.34439 \\
\hline 5 & STRIPPER & 63.19217 & 389.81920 & 0.18073 & -0.62437 & 0.65000 \\
\hline 6 & QH4 & 63.53974 & 388.61849 & 0.34756 & -1.20071 & 1.25000 \\
\hline 7 & $\mathrm{DH} 2$ & 64.08432 & 386.73711 & 0.54459 & -1.88139 & 1.95862 \\
\hline 8 & QV5 & 64.09559 & 384.87852 & 0.01127 & -1.85858 & 1.85862 \\
\hline 9 & DH3 & 64.11096 & 382.34482 & 0.01536 & -2.53370 & 2.53375 \\
\hline 10 & QH6 & 63.58923 & 380.45697 & -0.52173 & -1.88785 & 1.95862 \\
\hline 11 & QV7 & 63.08310 & 378.62562 & -0.50612 & -1.83135 & 1.90000 \\
\hline 12 & QH8 & 60.68570 & 369.95080 & -2.39740 & -8.67481 & 9.00000 \\
\hline 13 & DH4 & 60.35273 & 368.74597 & -0.33297 & -1.20483 & 1.25000 \\
\hline 14 & QV9 & 59.69557 & 366.51708 & -0.65716 & -2.22889 & 2.32375 \\
\hline 15 & QH10 & 58.85293 & 363.65910 & -0.84264 & -2.85798 & 2.97961 \\
\hline
\end{tabular}




$\begin{array}{lllllll}16 & \text { QV11 } & 56.98142 & 357.31152 & -1.87151 & -6.34759 & 6.61773 \\ 17 & \text { QH12 } & 55.68038 & 352.89879 & -1.30104 & -4.41272 & 4.60053 \\ 18 & \text { QV13 } & 54.16235 & 347.75007 & -1.51803 & -5.14871 & 5.36784 \\ & \text { QH14 } & 53.54456 & 345.65474 & -0.61778 & -2.09533 & 2.18451 \\ 20 & \text { DH5 } & 53.15435 & 344.33124 & -0.39021 & -1.32351 & 1.37983 \\ 21 & \text { QV15 } & 52.79025 & 341.85385 & -0.36410 & -2.47739 & 2.50400 \\ & \text { SEPTB1 } & 52.39096 & 339.13703 & -0.39929 & -2.71681 & 2.74600\end{array}$

\begin{tabular}{|c|c|c|c|c|c|}
\hline PUE_V.046 & 64.09782 & 384.51092 & $\begin{array}{l}0.00223 \\
\text { (From QV5) }\end{array}$ & -0.36761 & $\begin{array}{r}0.36761 \\
\text { (From QV5) }\end{array}$ \\
\hline XF059 & 63.39724 & 379.76229 & $\begin{array}{l}-0.19199 \\
\text { (From QH6) }\end{array}$ & -0.69468 & $\begin{array}{r}0.72073 \\
(\text { From QH6) }\end{array}$ \\
\hline MW0 60 & 63.27207 & 379.30937 & $\begin{array}{l}-0.31716 \\
\text { (From QH6) }\end{array}$ & -1.14761 & $\begin{array}{r}1.19063 \\
(\text { From } Q H 6)\end{array}$ \\
\hline MW125 & 57.35990 & 358.59521 & $\begin{array}{l}-1.49303 \\
\text { (From QH10) }\end{array}$ & -5.06389 & $\begin{array}{c}5.27940 \\
\text { (From QH10) }\end{array}$ \\
\hline DH127 & 57.20367 & 358.06531 & $\begin{array}{l}-1.64926 \\
\text { (From QH10) }\end{array}$ & $j^{-5.59379}$ & $\begin{array}{c}5.83185 \\
(\text { From QH10) }\end{array}$ \\
\hline PUE_V. 129 & 57.08538 & 357.66412 & $\begin{array}{l}0.10396 \\
(\text { From QV11) }\end{array}$ & 0.35261 & $\begin{array}{c}-0.36761 \\
\text { (From QV11) }\end{array}$ \\
\hline DV141 & 55.90263 & 353.65260 & $\begin{array}{l}-1.07879 \\
(\text { From QV11) }\end{array}$ & -3.65892 & $\begin{array}{c}3.81465 \\
\text { (From QV11) }\end{array}$ \\
\hline PUE_H. 143 & 55.78434 & 353.25140 & $\begin{array}{l}0.10396 \\
\text { (From QH12) }\end{array}$ & )$^{0.35261}$ & $\begin{array}{c}-0.36761 \\
\text { (From QH12) }\end{array}$ \\
\hline
\end{tabular}




\begin{tabular}{|c|c|c|c|c|c|}
\hline DH158 & 54.38460 & 348.50385 & $\begin{array}{l}0.22225 \\
\text { (From QV13) }\end{array}$ & 0.75380 & $\begin{array}{c}-0.78588 \\
(\text { From QV13) }\end{array}$ \\
\hline PUE_V. 160 & 54.26631 & 348.10268 & $\begin{array}{l}0.10389 \\
\text { (From QV13) }\end{array}$ & 0.35261 & $\begin{array}{c}-0.36761 \\
\text { (From QV13) }\end{array}$ \\
\hline MW1 66 & 53.92304 & 346.93843 & $\begin{array}{l}-0.23930 \\
\text { (From QV13) }\end{array}$ & -0.81164 & $\begin{array}{c}0.84618 \\
\text { (From QV13) }\end{array}$ \\
\hline DV168 & 53.76681 & 346.40854 & $\begin{array}{l}-0.39554 \\
\text { (From QV13) }\end{array}$ & -1.34154 & $\begin{array}{c}1.39863 \\
\text { (From QV13) }\end{array}$ \\
\hline PUE_H. 170 & 53.64853 & 346.00735 & $\begin{array}{l}0.10396 \\
(\text { From QH14) }\end{array}$ & 0.35261 & $\begin{array}{c}-0.36761 \\
\text { (From QH14) }\end{array}$ \\
\hline DV181 & 52.98505 & 343.17933 & $\begin{array}{l}-0.16930 \\
(\text { From DH5) }\end{array}$ & -1.15191 & $\begin{array}{r}1.16429 \\
\text { (From DH5) }\end{array}$ \\
\hline XF 183 & 52.91211 & 342.68301 & $\begin{array}{l}-0.24224 \\
\text { (From DH5) }\end{array}$ & -1.64823 & $\begin{array}{r}1.66594 \\
\text { (From DH5) }\end{array}$ \\
\hline
\end{tabular}

This table is a reproduction of Table 1, with all distances and coordinates converted to meters, by multiplying each value in Table 1 by 0.0254 .

\section{Reference:}

1. R. Thern, E. Auerbach, E. Bleser, M. Tanaka, 1985-1987 Horizontal Survey And Alignment, AGS/AD/Tech. Note No. 289, Brookhaven National Laboratory, Upton NY, 


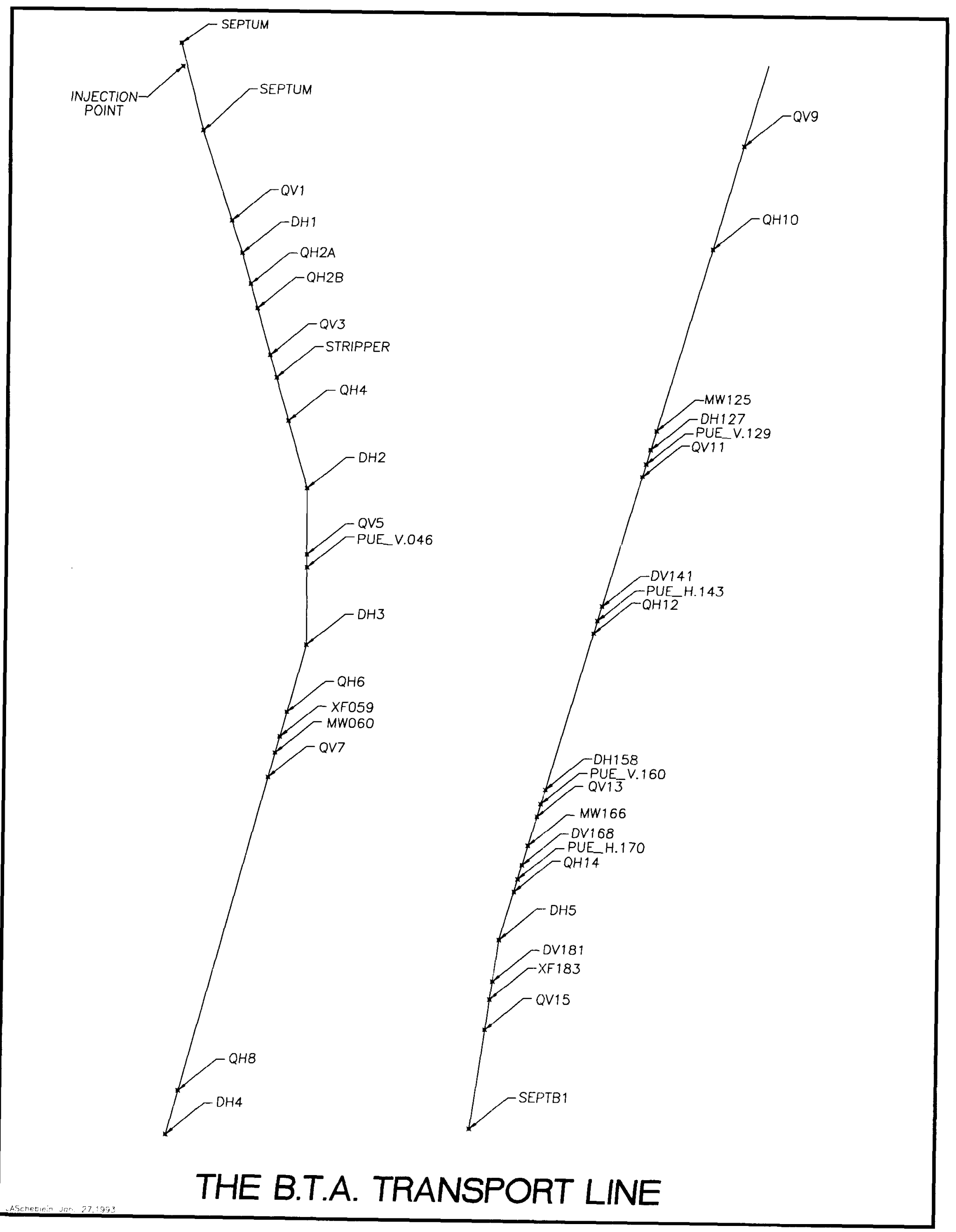

10 